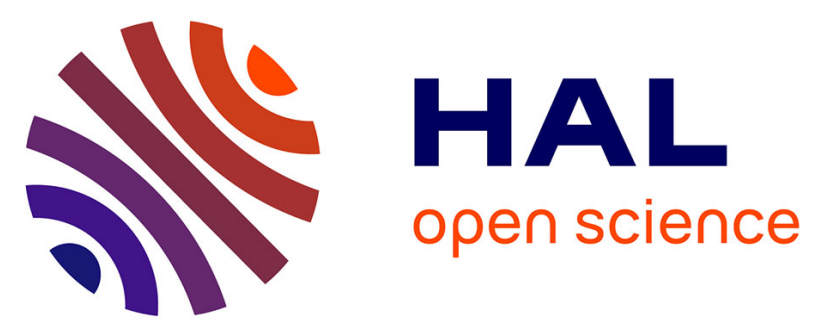

\title{
Near infrared emitting molecular rotor based on merocyanine for probing the viscosity of cellular lipid environments
}

Tarushyam Mukherjee, Ramon J Martinez-Sanchez, Kyong T Fam, Sophie Bou, Ludovic Richert, Delphine Garnier, Yves Mély, Sriram Kanvah, Andrey S Klymchenko, Mayeul Collot

\section{To cite this version:}

Tarushyam Mukherjee, Ramon J Martinez-Sanchez, Kyong T Fam, Sophie Bou, Ludovic Richert, et al.. Near infrared emitting molecular rotor based on merocyanine for probing the viscosity of cellular lipid environments. Materials Chemistry Frontiers, 2021, 5, pp.2459 - 2469. 10.1039/d0qm00872a . hal-03419791

\author{
HAL Id: hal-03419791 \\ https://hal.science/hal-03419791
}

Submitted on 10 Nov 2021

HAL is a multi-disciplinary open access archive for the deposit and dissemination of scientific research documents, whether they are published or not. The documents may come from teaching and research institutions in France or abroad, or from public or private research centers.
L'archive ouverte pluridisciplinaire HAL, est destinée au dépôt et à la diffusion de documents scientifiques de niveau recherche, publiés ou non, émanant des établissements d'enseignement et de recherche français ou étrangers, des laboratoires publics ou privés. 


\section{Journal Name}

\section{ARTICLE}

\section{Near Infrared Emitting Molecular Rotor Based on Merocyanine for Probing the Viscosity of Cellular Lipid Environments}

Received 00th January 20xx, Accepted 00th January 20xx

DOI: $10.1039 / x 0 x \times 00000 x$

www.rsc.org/

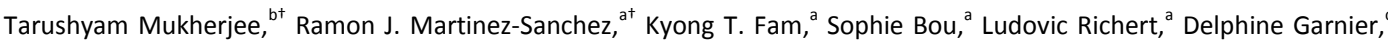 \\ Yves Mély, ${ }^{a}$ Sriram Kanvah, ${ }^{\text {b }}$ Andrey S. Klymchenko, ${ }^{a}$ Mayeul Collot ${ }^{{ }^{*}}$
}

\section{Introduction}

Among various biophysical parameters, the viscosity plays an important role in many biological processes. ${ }^{1}$ Indeed, the controlled diffusion of biomolecules is ensured by a defined viscosity. Therefore, variation of viscosity can be related to several diseases. 2, 3, 4 Fluorescence imaging, due to its high sensitivity and the relative affordability of microscopes has become a widespread bioimaging modality in laboratories. Consequently, many fluorescent probes sensitive to their molecular environment, ${ }^{5}$ have been developed to monitor intracellular variation of viscosity. Although different systems have been used to develop such probes including photoinduced electron transfer (PET) quenching, ${ }^{6}$ or organometallic complex, ${ }^{7}$ molecular rotors based on small fluorogenic organic molecules have proven their efficiency. ${ }^{8,9}$ 10, 11 Among the molecular rotors, those based on BODIPY benefit from a relatively high brightness due to high extinction coefficient and high quantum yields. ${ }^{12}$ Notably, they served to probe the viscosity of membranes, ${ }^{13,}{ }^{14}$ mitochondria, ${ }^{15}$ and

Laboratoire de Bioimagerie et Pathologies, UMR 7021, CNRS/Université de Strasbourg, 74 route du Rhin, 67401 Illkirch-Graffenstaden, France. E-mail: mayeul.collot@unistra.fr

Discipline of Chemistry, Indian Institute of Technology Gandhinagar, Palaj, Gandhinagar, Gujarat, India 382355

Laboratoire de Conception et Application des Molécules Bioactives (CAMB), UMR 7199, et Plateforme d'Analyse Chimique de Strasbourg-IIlkirch (PACSI), GDS 3670, CNRS/Université de Strasbourg, 74 route du Rhin, 67401 IIlkirch-Graffenstaden, France.

† These authors contributed equally to this work.

Electronic Supplementary Information (ESI) available: protocols, NMR and mass spectra, full photophysical characterisations, cytotoxicity assays. See

DOI: $10.1039 / \times 0 \times x 00000 x$ lysosomes. ${ }^{16}$ Their derivatives for Halo-tag protein labeling were also applied for organelle viscosity imaging. ${ }^{17}$ Moreover, we recently used a BODIPY rotor with a specific binder of phosphatidylserine as a efficient fluorogenic reporter of apoptosis. ${ }^{18}$ Despite the advantageous properties of BODIPYbased rotor, the latter remain limited to the green color (emission around $500 \mathrm{~nm}$ ) thus preventing its use in multicolor imaging in combination with the widely used GFP or other common green emitting dyes. For these reasons, a special attention has been drawn towards the development of redshifted molecular rotors. Among them, those emitting in the near infrared (NIR) region possess multiple advantages including, reduced background noise from cell autofluorescence, limited photo-damage and compatibility with other dyes emitting in visible range for multicolor imaging. Moreover, due to deep tissue penetration and reduced light scattering NIR probes are adapted for in vivo imaging. ${ }^{19-20}$ NIR rotors based on cationic styryl dyes possess interesting viscosity response but are limited by rather low brightness and broad spectra. ${ }^{21}, 22,23,24,25,26$ Although rotors based on carbocyanines display enhanced brightness, ${ }^{27,} 28$ they remain cationic and thus tend to target the mitochondria which limits their versatility. To circumvent this issue, red-shifted neutral rotors have been developed. ${ }^{29}$ Surprisingly, only few of them, mainly carbocyanine-based merocyanine, emitting in the NIR have been described in the literature. ${ }^{30,} 31$ Recently dihydroxyxanthene hemicyanines have emerged as a new class of efficient cationic fluorophores. ${ }^{32}$ 


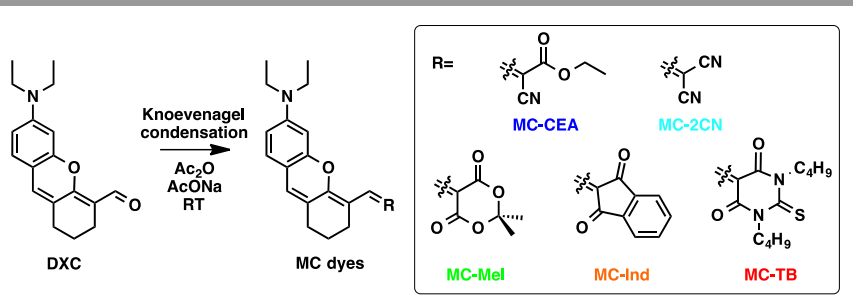

Scheme 1. Synthesis of MC dyes.

The latter emit in the NIR region with narrow peaks combined with important brightness making them amenable to in vivo imaging. ${ }^{33}, 34,35$ Upon adapted modification, caging and functionalization, various probes were successfully obtained including probes for $\mathrm{pH}^{36,37}$ hypoxia, ${ }^{33}$ hydrazine, ${ }^{38}$ enzyme activity, $^{39,} 40$ and calcium. ${ }^{41}$ The dihydroxyxanthene hemicyanines can be obtained from Knoevenagel condensations of cationic acids on dihydro-xanthene carbaldehyde (DXC, scheme 1). ${ }^{42,43,44}$ Interestingly, DXC is, by itself, emissive in the visible range and was used as a red/green ratiometric $\mathrm{pH}$ probe. ${ }^{45}$ Herein, we investigated on the ability of the extended DXC scaffold to provide noncharged merocyanines with desirable photophysical and molecular rotor properties. When studying the five new fluorescent probes (MC), notable differences were observed and a structure/properties relationship has been established. Among the new MCs, MC-TB displayed a high sensitivity to viscosity with reduced sensitivity to polarity, high brightness and photostability combined with a fluorescence emission in the NIR (700 nm) with sharp absorption and emission peaks. In cells, MC-TB successfully probed LDs and was additionally transformed into a mitochondrial probe that reported on heterogeneous mitochondrial viscosity using confocal microscopy and fluorescence lifetime imaging (FLIM).

\section{Results \& discussion}

\section{Synthesis}

The synthesis of the merocyanines (MCs) arose from a Knoevenagel condensation between 5 different non-cationic acidic C-H compounds and 6-(Diethylamino)-2,3-dihydro-1Hxanthene-4-carbaldehyde (DXC, scheme 1). The latter was obtained following the protocol described by Romieu et $a .^{42}$ with an adapted purification method (See SI). While malononitrile (2CN) and cyano-ethylacetate (CEA) were chosen as typical molecular rotor blocks, ${ }^{11}$ Meldrum's acid (Mel) and indane-1,3-dione (Ind) were chosen as efficient cyclic Knoevenagel donors. Thiobarbituric acid (TB), which is a part of the commercially available merocyanine MC- $540,{ }^{46}$ was also used. Screening conditions for the Knoevenagel condensation using piperidine as a usual catalyst in various solvents led to either low conversions or degradation. Finally, acetic anhydride in the presence of sodium acetate at room temperature provided the desired compounds in modest to high yields (up to $88 \%$ ). The obtained compounds were characterized by ${ }^{1} \mathrm{H},{ }^{13} \mathrm{C} N M R$ and high-resolution mass spectroscopy (See $\mathrm{SI}$ ). In the case of MC-CEA, two isomers could have been obtained. However, further NMR studies including NOESY and ${ }^{13} \mathrm{C}-{ }^{1} \mathrm{H}$ coupled NMR unambiguously confirmed that the $E$-isomer was selectively obtained (See SI).
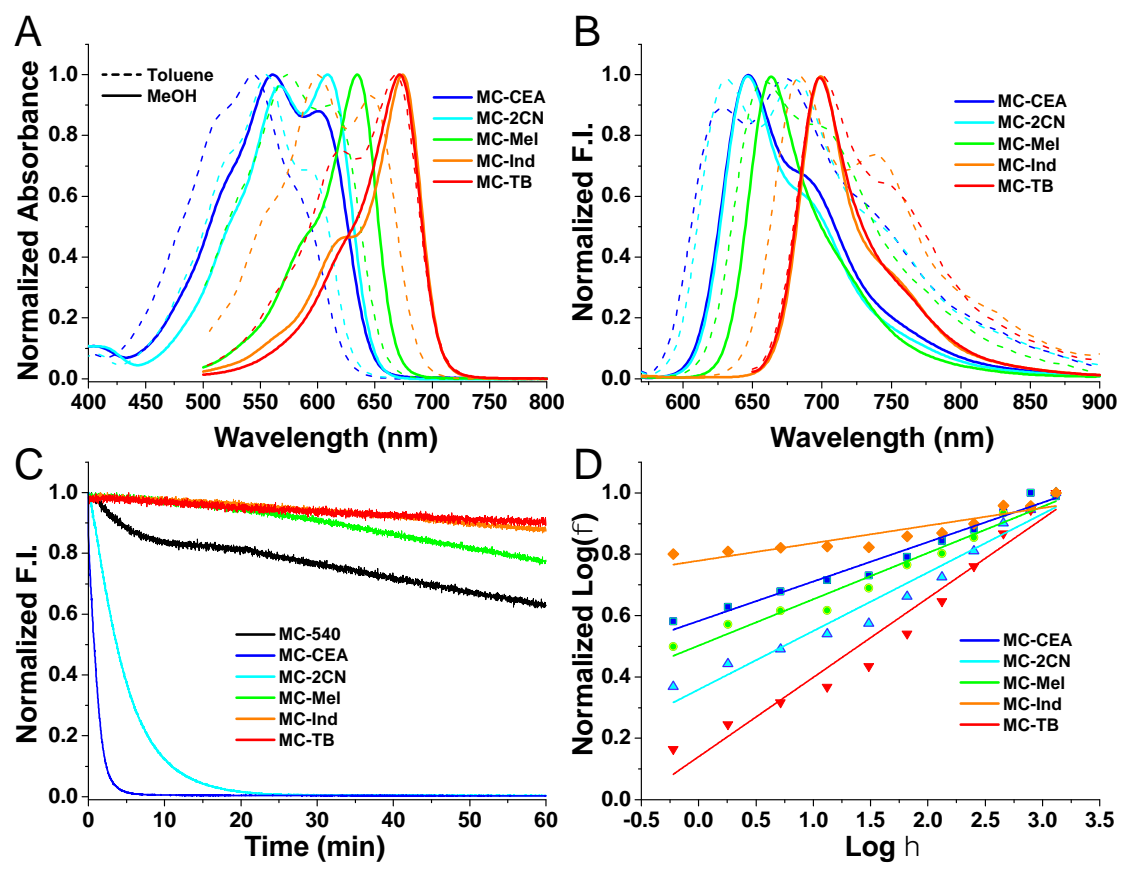

Figure 1. Normalized absorption (A) and emission (B) spectra of MCs (5 $\mu \mathrm{M})$ in toluene (Dash lines) and methanol (Solid lines). (C) Fluorescence intensity of MCs (5 $\mu \mathrm{m}$ in DMSO) monitored under continuous illumination. The dyes were all excited at a wavelength corresponding to extinction coefficient of $20,000 \mathrm{M}^{-1} . \mathrm{cm}^{-1}$. (D) Quantum yield $(\phi)$ enhancement of MCs upon increase of the viscosity using $\mathrm{MeOH} / \mathrm{glycerol}$ mixtures. $\eta$ is the viscosity of the MeOH/Glycerol mixtures expressed in mPa.s. 


\section{Journal Name}

\section{ARTICLE}

Table 1. Photophysical properties of the merocyanines at $5 \mu \mathrm{M}$ concentration.

\begin{tabular}{|c|c|c|c|c|c|c|c|c|c|}
\hline Dye & Solvent & $\begin{array}{l}\text { Abs } \max \\
(\mathrm{nm})\end{array}$ & $\left(\mathbf{M}^{-1} \cdot \mathrm{cm}^{-1}\right)$ & $\begin{array}{c}\mathrm{FWH}^{\mathrm{a}}{ }_{\mathrm{Abs}} \\
(\mathrm{nm})\end{array}$ & $\begin{array}{l}\mathrm{Em} \max \\
(\mathrm{nm})\end{array}$ & $\begin{array}{c}\mathrm{FWHM}{ }_{\mathrm{Em}}^{\mathrm{a}} \\
(\mathrm{nm})\end{array}$ & $\begin{array}{c}\text { Stokes Shift } \\
(\mathrm{nm})\end{array}$ & & $\begin{array}{c}\text { Shift }^{\mathrm{b}} \\
\text { (Abs/Em) }\end{array}$ \\
\hline \multirow{2}{*}{ MC-CEA } & Toluene & 544 & 32,600 & 115 & 675 & 138 & 131 & 0.007 & \multirow{2}{*}{$47 / 27^{c}$} \\
\hline & $\mathrm{MeOH}$ & 591 & 37,500 & 120 & 648 & 83 & 57 & 0.07 & \\
\hline \multirow{2}{*}{ MC-2CN } & Toluene & 555 & 42,800 & 108 & 681 & 130 & 126 & 0.004 & \multirow{2}{*}{$54 / 35^{\mathrm{c}}$} \\
\hline & $\mathrm{MeOH}$ & 609 & 57,800 & 109 & 646 & 76 & 37 & 0.03 & \\
\hline \multirow{2}{*}{ MC-Mel } & Toluene & 574 & 40,000 & 106 & 657 & 102 & 83 & 0.003 & \multirow{2}{*}{$61 / 7$} \\
\hline & $\mathrm{MeOH}$ & 635 & 86,400 & 57 & 664 & 54 & 29 & 0.05 & \\
\hline \multirow{2}{*}{ MC-Ind } & Toluene & 600 & 48,800 & 119 & 684 & 104 & 84 & 0.006 & \multirow{2}{*}{$74 / 15$} \\
\hline & $\mathrm{MeOH}$ & 674 & 106,600 & 52 & 699 & 42 & 25 & 0.08 & \\
\hline \multirow{2}{*}{ MC-TB } & Toluene & 668 & 74,600 & 97 & 701 & 90 & 33 & 0.005 & \multirow{2}{*}{$4 / 3^{c}$} \\
\hline & $\mathrm{MeOH}$ & 672 & 106,000 & 59 & 698 & 49 & 26 & 0.01 & \\
\hline
\end{tabular}

${ }^{a}$ FWHM: Full Width at Half Maximum, which denotes the broadness of the peak

${ }^{\mathrm{b}}$ Solvatochromic shift between toluene and $\mathrm{MeOH}$ expressed in $\mathrm{nm}$

${ }^{\mathrm{c}}$ Negative solvatochromism in emission

\section{Spectroscopic studies}

First, absorption and emission spectra of MCs were recorded in five different solvents with increasing polarity (Figure 1A, 1B \& S1). Their photo-physical properties in non-polar toluene and polar methanol are reported in table 1 (for other solvents see tables S1-S5). It was first noted that all MCs emitted in the NIR range with $\lambda_{E m}$ ranging from 646 to $701 \mathrm{~nm}$. In non-polar solvents (toluene, dioxane), all the dyes displayed multiple peaks and broad bands in both absorbance and emission spectra, denoting the potential presence of different vibrational states that can be assigned to rotations around the $\sigma$ bonds. ${ }^{9}, 47$ In those solvents the emission spectra are different from the mirror image of the absorption spectra, indicating that the emissive species at the excited state are different from those in the ground state, which is typical for push-pull fluorophores. In polar solvents (ACN, DMSO and $\mathrm{MeOH}$ ) extinction coefficients and quantum yields significantly increased (Figure S2, tables 1 \& S1-S5), and absorption and emission peaks sharpened (Figure S1), which is in line with previous works on neutral merocyanines. ${ }^{47}$ Additionally, superimposition of absorption and excitation spectra in both toluene and methanol indicated that the entire absorption spectra correspond to emissive species (Figure S3).

Interestingly, a structure/properties relationship can be established depending on the nature of substituents connected to the DDX scaffold ( $R$ in scheme 1). Indeed, it was noteworthy that MCs displayed significant differences of their photo-physical properties between those bearing "open" form substituents (MC-CEA and MC-2CN) and those bearing "cyclic" ones (MC-Mel, MC-Ind and MC-TB). In polar solvents, the "cyclic" MCs displayed sharp peaks in both absorption and emission, which are mirror images, indicating similar geometries in both $\mathrm{S}_{0}$ and $\mathrm{S}_{1}$ states. Conversely, the "open" MCs, conserved broad and multi-peak spectra (Figure S4) along with limited extinction coefficients from 32,600 to $57,800 \mathrm{M}^{-1} \cdot \mathrm{cm}^{-1}$ and large Stokes' shifts (table S1-S5). These observations are consistent with the structures as cyclic substituents restrict the rotation around the $\sigma$ bonds in both ground and excited states, thus limiting the number of vibrational states (Figure S4). As a consequence of the narrowing of the peaks, the "cyclic" MCs also showed much higher extinction coefficients (up to 106,000 $\mathrm{M}^{-1} \cdot \mathrm{cm}^{-1}$ ). Another difference arose from the solvatochromism properties. While the "open" MCs displayed a relatively large negative solvatochromism in emission, "cyclic" ones displayed notable positive solvatochromism in absorption and small positive one in emission (MC-Mel and MC-Ind) or, in the case of MC-TB, virtually no solvatochromism (Table 1 ). The most striking difference between the "open" and "cyclic" MCs was observed in the photostability. MCs along with MC-540 (a reference and commercial merocyanine ${ }^{46}$ ) were irradiated at the same value of extinction coefficient for $1 \mathrm{~h}$ (Figure 1C). The results showed that whereas the "open" MCs were totally photobleached within $20 \mathrm{~min}$, the "cyclic" MCs displayed 
higher photostability, also compared to MC-540, with only $22 \%$ (MC-Mel), 11\% (MC-Ind) and 7\% (MC-TB) loss of their fluorescence intensity after $1 \mathrm{~h}$ of irradiation.

Overall the MCs possessed low quantum yields in solvents ranging from 0.001 (MC-TB in acetonitrile) to 0.08 (MC-Ind in $\mathrm{MeOH}$ ) making them potentially efficient molecular rotors. Consequently, the quantum yield enhancement of MCs upon increase of the viscosity was measured (Figure 1D, for absorption and emission spectra see S5-S9). Except MC-Ind, the MCs showed notable quantum yield enhancement with increase in viscosity. The enhancement varied from 3-fold (MC-CEA) to 20-fold (MC-TB) with quantum yields in glycerol ranging from (0.11 to 0.29). Once again, a structure/activity relationship can be established, as it appeared that MCs bearing electron-withdrawing substituents exhibited enhanced sensitivity to the viscosity. Indeed, MC-TB bearing an electronwithdrawing thiobarbituryl group displayed higher rotor effect than MC-Mel bearing esters and MC-Ind bearing ketones being the less responsive to viscosity. In the light of these results, it appeared that MC-TB displayed several appealing properties as a fluorogenic molecular rotor suitable in bioimaging. 1) MC-TB presented the lowest solvatochromism in solvents (table 1 ), the lowest basal quantum yield in methanol along with the highest sensitivity to the viscosity (quantum yield increased 20 -fold from $\mathrm{MeOH} \phi=0.01$ to glycerol $\phi=0.20$ ). 2) It possessed the highest extinction coefficient up to $106,000 \mathrm{M}$ ${ }^{1} . \mathrm{cm}^{-1}$ with sharp absorption and emission spectra. 3) MC-TB proved to be the most photostable. 4) Finally, MC-TB is the most red-shifted MCs, being efficiently excited at $640 \mathrm{~nm}$ (widely used laser line) and emitting in the NIR ( 700 nm). Prior to imaging, MTT assays were performed on MCs, showing no cytotoxicity of MC-TB up to $5 \mu \mathrm{M}$ (Figure S10).

\section{Probing lipid droplets}
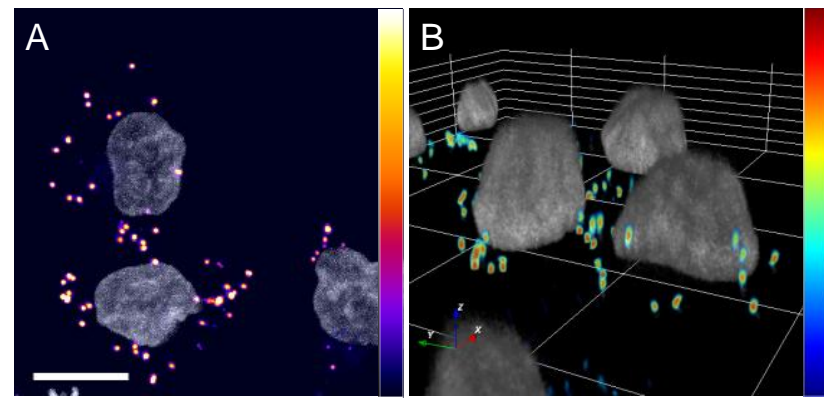

Figure 2. Fluorescence imaging of LDs using MC-TB. (A) Max Z-projection and (B) 3D images of KB cells incubated with MC-TB $(1 \mu \mathrm{M})$. Scale bar is $15 \mu \mathrm{m}$ in (A). Squares in xy are $15 \times 15 \mu \mathrm{m}$, graduation in z scale is $2 \mu \mathrm{m}$ in (B). Colored spots are LDs, the color scales of fluorescence intensity are indicated on the right. The nuclei are in grey.

Based on our experience in the field, ${ }^{48,49,50,51}$ and due to the fluorogenic properties of MC-TB in viscous media combined to its lipophilic nature (non-charged structure and presence of two n-butyl groups), MC-TB was expected to act as an efficient probe for lipid droplets (LDs). Indeed, LDs are viscous ${ }^{52}$ and highly hydrophobic organelles. ${ }^{50}$ Whereas MC-TB is nonfluorescent in PBS due to aggregation caused quenching suggested by its broad absorption spectrum (Figure S11), it displays a sharp emission $(\phi=0.15)$ in the viscous vitamin $E$ acetate oil, ${ }^{53}$ thus provoking a fluorescence enhancement of 860 -fold at $696 \mathrm{~nm}$. In order to confirm its performance in bioimaging, MC-TB $(1 \mu \mathrm{M})$ was incubated with KB cells and imaged by laser confocal microscopy using a near-infrared detection channel (excitation wavelength at $640 \mathrm{~nm}$ ). Thanks to its brightness and photostability, MC-TB revealed the cell LDs as intense spots in 3D images obtained from $>50$ images $Z$ stack (Figure 2). Since LDs are already highly viscous, we wanted to prove that the newly developed rotor could image variations of viscosity in a different specifically-targeted organelle.

\section{Synthesis of a viscosity probe for mitochondria}

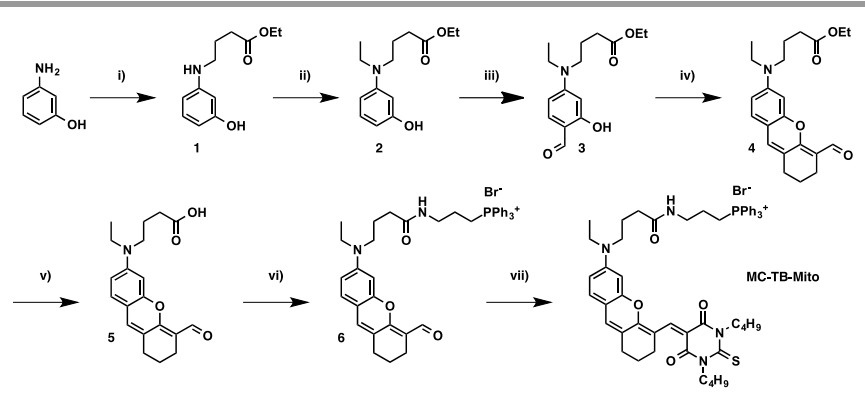

Scheme 2. Synthesis of MC-TB-Mito. i) Ethyl 4-bromobutyrate, $\mathrm{NaHCO}_{3}, \mathrm{KI}, \mathrm{DMSO}$. ii) Ethyl iodide, $\mathrm{NaHCO}_{3}$, DMSO. iii) $\mathrm{POCl}_{3}, \mathrm{DMF}$. iv) 2-bromocyclohex-1-ene-1carbaldehyde, $\mathrm{Cs}_{2} \mathrm{CO}_{3}$, DMF. v) $\mathrm{NaOH}$ (aq), $\mathrm{MeOH}$. vi) (3-ammoniopropyl) triphenylphosphonium dibromide, HATU, DIEA, DMF. vii) 1,3-di-n-butyl-2-thiobarbituric acid, $\mathrm{Ac}_{2} \mathrm{O}$, AcONa.

Monitoring mitochondrial viscosity gained attention as it revealed important information about cellular biology and provided new insights into certain diseases. ${ }^{54,55,56}$ In order to demonstrate the versatility of our NIR rotor, we undertook the synthesis of a functionalized form of MC-TB that would target the mitochondria, namely MC-TB-Mito (Scheme 2 ). To achieve this aim, a functional arm was installed in the first step as an ester during the $\mathrm{N}$-alkylation of 3-hydroxyaniline. The aniline 1 was further $\mathrm{N}$-alkylated and the obtained compound 2 was then formylated by Vilsmeier-Haack reaction, yielding aldehyde 3. The DCX derivative 4 was then obtained and the ester was saponified to afford acid 5 . The latter was coupled to an amine bearing a triphenylphosphonium moiety as a specific mitochondrial targeting moiety. ${ }^{57}$ Aldehyde 6 was then involved in a Knoevenagel reaction with 1,3-di-n-butyl-2thiobarbituric acid to finally afford MC-TB-Mito.

Once the probe obtained, spectroscopic studies were performed. Although MC-TB-Mito displayed similar photophysical properties to its parent MC-TB dye, it possessed higher quantum yield in methanol ( 0.05 and 0.01 respectively). The probe responded in a fluorogenic manner to the increase of viscosity with a fluorescence enhancement of 9.4-fold at $700 \mathrm{~nm}$ (figure $3 \mathrm{~A}$ ), reaching a maximum quantum yield of 0.43 in glycerol. Following the Förster-Hoffmann equation, an excellent correlation was found between MC-TB-Mito's quantum yield and the viscosity (Figure $3 \mathrm{~B}$ ). Finally, the mean fluorescence lifetime of MC-TB-Mito was measured in solutions with increasing viscosity. The fluorescence decay was 
shown to be monoexponential in low viscosity media and biexponential when the glycerol content reached $40 \%$ (table S6) with a gradual increase from 0.16 ns in low viscosity solvent $(\mathrm{MeOH})$ to $1.97 \mathrm{~ns}$ in highly viscous glycerol (Figure
3C). Overall, MC-TB-Mito showed high sensitivity to the viscosity combined with a high brightness in the NIR region making it a promising mitochondrial viscosity probe.
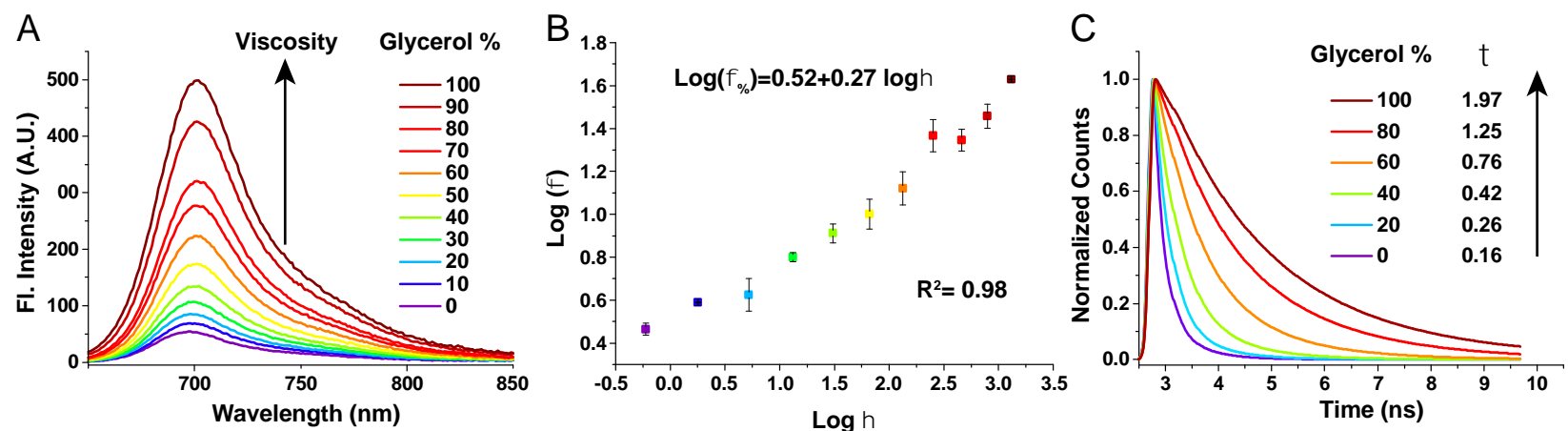

Figure 3. Spectroscopic study of MC-TB-Mito. (A) Emission spectra of MC-TB-Mito in various solutions of glycerol/MeOH mixture, the spectra were normalized to their absorption at $640 \mathrm{~nm}$, to reflect the variation in the fluorescence quantum yield. (B) Correlation between the fluorescence quantum yield of MC-TB-Mito and the viscosity of the medium using $\mathrm{MeOH} /$ glycerol mixtures. (C) Effect of viscosity on the lifetime decay of MC-TB-Mito. $\tau$ is the mean fluorescence lifetime. Concentration of MC-TB-Mito was $1 \mu \mathrm{M}$. Excitation wavelength was $640 \mathrm{~nm} . \eta$ is the viscosity of the $\mathrm{MeOH} / \mathrm{Glycerol}$ mixtures expressed in mPa.s.

\section{Cellular imaging}

At first, the cytotoxicity of MC-TB-Mito was assessed by MTT assay. Although MC-TB-Mito was toxic for cells at high concentration $(5 \mu \mathrm{M})$, the cell viability at the concentration used in cellular imaging ( $1 \mu \mathrm{M})$ was comparable to control cells (Figure S12). In a second time, we determined the subcellular localization of MC-TB-Mito using laser scanning confocal microscopy (Figure 4A-C). Images of HeLa cells co-stained with MitoTracker green and MC-TB-Mito exhibited a high colocalization pattern with a Pearson's coefficient of $0.82 \pm$ 0.03 (12 images), thus confirming that MC-TB-Mito targeted the mitochondria. Interestingly, a difference was observed between the fluorescence staining of mitochondria with $\mathrm{MC}$ TB-Mito and MitoTracker green. The intensity of MC-TB-Mito green was less homogeneous within the cells compared MitoTracker, suggesting that the viscosity of mitochondria could differ between cells. Thereafter, we focused on evaluating the ability of MC-TB-Mito to probe variations of mitochondrial viscosity (Figure 4D-F). To this aim, we used nystatin, a drug known to increase the viscosity of mitochondria. ${ }^{58}$ Non-treated HeLa cells and cells pre-treated with $5 \mu \mathrm{M}$ nystatin were incubated with MC-TB-Mito and were submitted to live cell imaging. Confocal images revealed a notable increase of the NIR fluorescence intensity (3.7-fold) when the cells were treated with nystatin, thus confirming the high sensitivity of MC-TB-Mito and its ability to sense mitochondrial viscosity. Once again, in normal conditions, notable differences in fluorescence intensity were observed between cells of a same sample (Figure S13). Taking advantage of the fluorescence lifetime dependence on the viscosity, fluorescence lifetime imaging (FLIM) was performed on the same non-treated and treated cells. Although the probe spanned its lifetime from 0.16 to $1.97 \mathrm{~ns}$ in cuvette, only small non-significant differences of mean lifetime were observed between the two conditions (see figure S14, figure $4 \mathrm{G}$ mean $\tau=$ $1.56 \pm 0.028 \mathrm{~ns}$, Figure $4 \mathrm{H}$ mean $\tau=1.66 \pm 0.054 \mathrm{~ns})$. The explanation is connected to the correlation between the fluorescence intensity and the fluorescence lifetime. Indeed, in absence of fluorescence staining that is non-dependent on viscosity, and when performing imaging in normal conditions, only cells displaying sufficiently bright mitochondria were visible and have been imaged by FLIM. Consequently, all imaged cells displayed relative high fluorescence lifetime values. In absence of nystatin, FLIM not only revealed heterogeneity of lifetime within a cell (Figure 4G) but also between two adjacent cells (Figure 4I), thus confirming our observation in intensiometric images (Figure S13). In order to better understand the heterogeneity within the cells of a same population, we hypothesized that bright cells, with high mitochondrial viscosity, could be assigned to an apoptotic status. To verify this hypothesis, apoptosis was induced by staurosporine ${ }^{59,60}(1 \mu \mathrm{M})$ and then cells were incubated with MC-TB-Mito and imaged with confocal microscope. When comparing to control cells (Figure 5A), the apoptotic cells displayed a high mitochondrial fluorescence signal (Figure 5B) with a similar average intensity than those treated with nystatin (Figure 4F). Although this observation could be assigned to a variation of mitochondrial membrane's potential during apoptosis thus affecting the accumulation of the probe, our results are in line with previous works that showed that apoptosis was associated to an increase of mitochondrial viscosity. ${ }^{26,61,62}$ 


\section{Journal Name}

\section{ARTICLE}
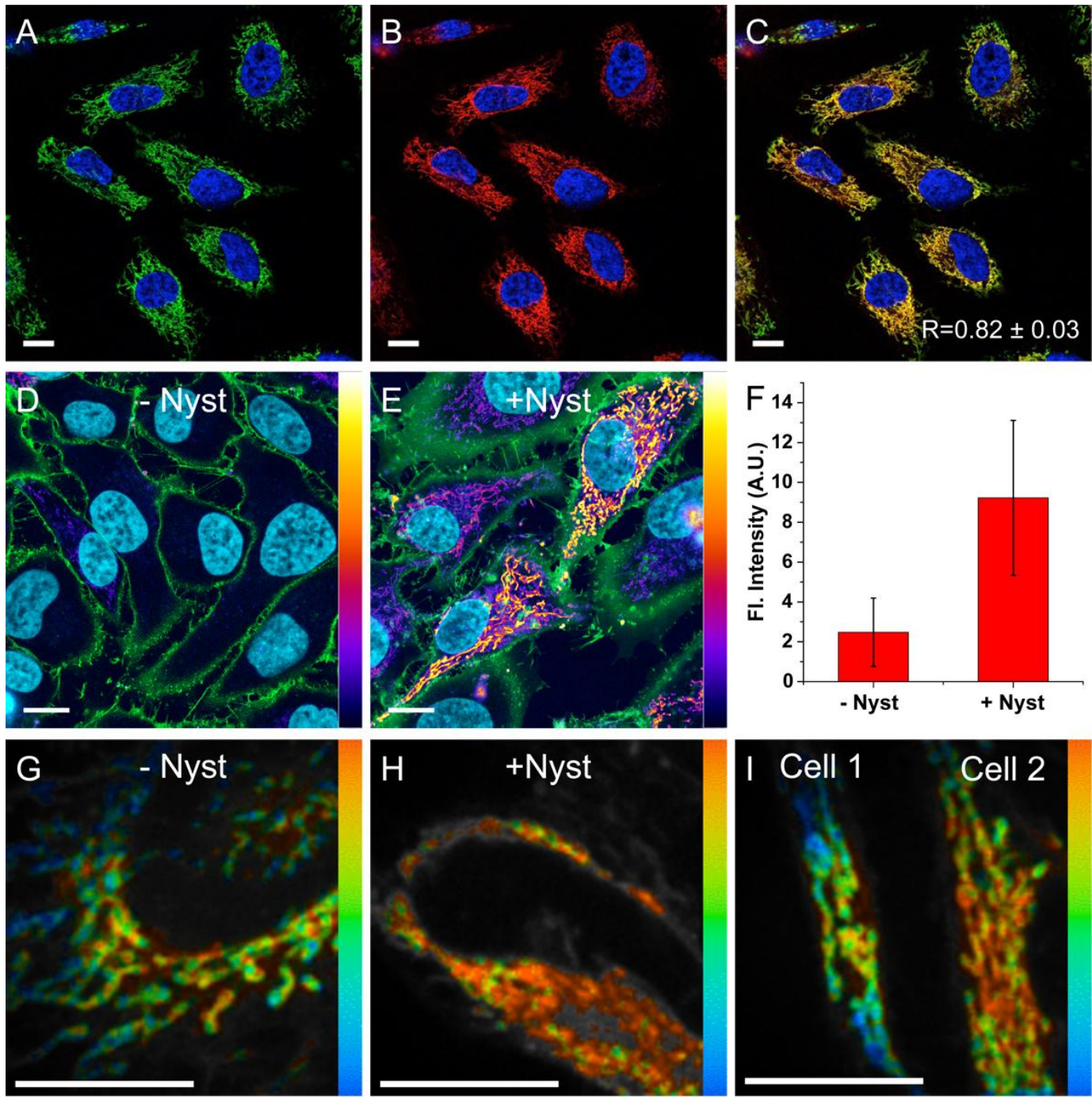

Figure 4. Fluorescence imaging of live cell's mitochondria using MC-TB-Mito. Colocalization study: HeLa cells stained with Hoechst (blue color, $5 \mu$ g.mL ${ }^{-1}$ ) and with (A) MitoTracker green (green, $200 \mathrm{nM}$ ) and (B) MC-TB-Mito (red, $1 \mu \mathrm{M}$ ), (C) is the merge of (A) and (B), R is the Pearson's coefficient of colocalization. HeLa cells stained with Hoechst (cyan, 5 $\mu \mathrm{g} \cdot \mathrm{mL}^{-1}$ ), MemBright-488 (green, $200 \mathrm{nM}$ ) and MC-TB-Mito (color-coded intensity, $1 \mu \mathrm{M}$ ) in normal condition (- Nyst, D) and after incubation with $5 \mu \mathrm{M}$ nystatin (+ Nyst, E). (F) is the average fluorescence intensity of cells in (D) and (E). T-test value showed significant difference $\left(p=3.5 \times 10^{-21}, N=50\right)$. FLIM images of HeLa cells stained with MC-TB-Mito (1 $\left.\mu M\right)$ in $(\mathrm{G})$ normal condition and $(\mathrm{H})$ after incubation with nystatin $(5 \mu \mathrm{M})$. The FLIM color code ranges from $1.42 \mathrm{~ns}$ to $1.64 \mathrm{~ns}$. (I) FLIM images showing two individual adjacent cells in normal conditions displaying difference of mean fluorescence lifetime. Scale bars are $15 \mu \mathrm{m}$. The color bar indicates the color lookup table used.

\section{Conclusion}

To fill the gap in development of non-cationic and bright NIR molecular rotors, we undertook the synthesis of five new NIRemitting fluorophores based on the dihydro-xanthene scaffold. The spectroscopic studies showed significant differences in their photophysical properties and structure/properties relationships have been established, which can assist chemists in developing new molecular rotors with improved properties. Among the developed MCs, MC-TB possessed appealing properties for monitoring viscous lipid environments as it showed a high rotor effect from methanol to glycerol displaying high brightness in viscous medium. When incubated with cells, MC-TB efficiently revealed the most hydrophobic and viscous organelles namely the lipid droplets. To demonstrate the versatility of the new rotor, we synthesized MC-TB-Mito that targeted the mitochondria. The probe 
displayed high sensitivity to the viscosity with 9.4-fold fluorescence enhancement with a significant increase of mean fluorescence lifetime (from 0.16 ns to $1.97 \mathrm{~ns}$ ). MC-TB-Mito efficiently reported on variations of mitochondrial viscosity using both intensiometric imaging and FLIM. In normal conditions, heterogeneity in mitochondrial viscosity between cells of the same population was observed. We thus hypothesized that it could be assigned to different apoptotic status that can alter the physiological functions of mitochondria. Indeed, further cell imaging using induction of apoptosis confirmed a correlation between the apoptosis and the increase of mitochondrial viscosity. We believe that the impressive performances of this newly developed NIR rotor will inspire further development of red shifted merocyaninebased rotors finding their use in multicolor imaging and potentially in vivo imaging.
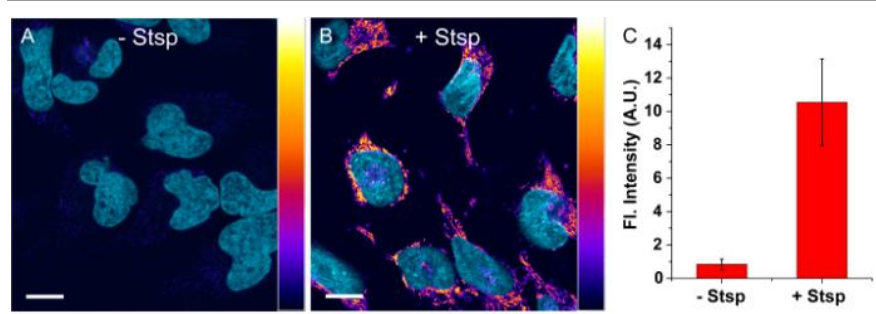

Figure 5. Laser scanning confocal microscopy images of HeLa cells stained with MC-TBMito $(1 \mu \mathrm{M})$ without staurosporine (- Stsp, A) and with $1 \mu \mathrm{M}$ staurosporine (+ Stsp, B) (C) is the average of cells' fluorescence intensity. T-test value showed significant difference $\left(p=3.5 \times 10^{-43}, \mathrm{~N}=50\right)$.

\section{Experimental section}

\section{Materials}

The solvents were of analytical grade and dry, reagents and starting materials were purchased form $\mathrm{TCl}$, Alfa Aesar or Merck Millipore. Nystatin was purchased from Merck. Water was miliQ water. Phosphate buffered saline (PBS) was from Eurobio (Gourtaboeuf, France) and fetal bovine serum (FBS) from Lonza.

\section{Synthesis}

Protocols and characterizations can be found in the supplementary information. NMR spectra were recorded on a Bruker Avance III $400 \mathrm{MHz}$ or $500 \mathrm{MHz}$ spectrometers. Mass spectra were obtained using an Agilent Q-TOF 6520 mass spectrometer. LCs were performed on Agilent 1200 series.

\section{Spectroscopic studies}

All the solvents were spectroscopic grade. Absorption and emission spectra were recorded on a Cary 400 Scan ultraviolet-visible spectrophotometer (Varian) and a FluoroMax-4 spectrofluorometer (Horiba Jobin Yvon) respectively. For standard recording of fluorescence spectra, the emission was collected $10 \mathrm{~nm}$ after the excitation wavelength. All the spectra were corrected from wavelengthdependent response of the detector. Quantum yields were determined by comparison with Cresyl violet in $\mathrm{MeOH}(\phi=$
$0.53)^{63}$ or Rhodamine 800 in $\mathrm{EtOH}(\phi=0.25)^{64}$ as references using the following equation:

$$
\mathrm{QY}=\mathrm{QY}_{R} \times \frac{I \times O D_{R} \times n^{2}}{I_{R} \times O D \times n_{R}^{2}}
$$

where $Q Y$ is the quantum yield, I is the integrated fluorescence intensity, $\mathrm{n}$ is the refractive index, and OD is the optical density at the excitation wavelength. $R$ represents the reference. The glycerol-containing solutions were placed in Eppendorfs, stirred and warmed up at $60^{\circ} \mathrm{C}$ for an optimal homogenous before being transferred in cuvettes, cooled at room temperature and measured. The viscosity of the $\mathrm{MeOH} /$ glycerol mixtures varies from 0.6 to $1317 \mathrm{mPa} . \mathrm{s}^{29}$

\section{Photostability assay}

Solutions of fluorophores were placed in a $50 \mu \mathrm{L}$ quartz cuvette and the entire solution was continuously irradiated for $1 \mathrm{~h}$ at an excitation wavelength corresponding to an extinction coefficient of $20,000 \mathrm{M}^{-1} . \mathrm{cm}^{-1}$ for the different dyes with excitation slit opening at $16 \mathrm{~nm}$. The fluorescence intensity was monitored at the maximum emission wavelength determined by the emission spectra prior to kinetic measurements. Emission slit opening was set to obtain 1,000,000 A.U.

\section{Lifetime}

Time-resolved fluorescence measurements were performed with the time-correlated, single-photon counting technique using the excitation pulses at $682 \mathrm{~nm}$ provided by a pulsepicked supercontinuum laser (EXR-20, NKT Photonics) equipped with an UV extension (SuperK EXTEND-UV). The emission was collected through a polarizer set at the magic angle and a $16 \mathrm{~nm}$ band-pass monochromator ( $\mathrm{H} 10$, JobinYvon) at the maximum of fluorophore emission. The instrumental response function was recorded with a polished aluminium reflector, and its full-width at half-maximum was 50 ps. Time-resolved intensity data were treated with an analysis using a Matlab home-made software based of Tcspcfit ${ }^{65}$ and the Maximum Entropy Method (MEM). ${ }^{66} 67$ In all cases, the $\chi^{2}$ values were close to 1 , and the weighted residuals as well as the autocorrelation of the residuals were distributed randomly around 0 , indicating an optimal fit. Rhodamine 800 was used as a reference (1.93 ns in ethanol). ${ }^{64}$

\section{Cytotoxicity}

Cytotoxicity of MCs was evaluated by MTT assay (3-(4,5dimethylthiazol-2-yl)-2,5-diphenyltetrazolium bromide). A total of $1 \times 10^{4} \mathrm{~KB}$ cells/well were seeded in a 96-well plate $18 \mathrm{~h}$ prior to the experiment in Dulbecco's Modified Eagle Medium (DMEM, Gibco Lifetechnologies) complemented with 10\% FBS, Gentamicin $(100 \mu \mathrm{g} / \mathrm{mL})$, L-Glutamine $(2 \mathrm{mM})$, non-essential amino acids $(1 \mathrm{mM}), \mathrm{MEM}$ vitamin solution (1\%) and were incubated in a $5 \% \mathrm{CO}_{2}$ incubator at $37^{\circ} \mathrm{C}$. After medium 
removal, an amount of $100 \mu \mathrm{L}$ DMEM containing $5 \mu \mathrm{M}, 1 \mu \mathrm{M}$ or $0.2 \mu \mathrm{M}$ of MCs was added to the KB cells and incubated for $24 \mathrm{~h}$ at $37^{\circ} \mathrm{C}\left(5 \% \mathrm{CO}_{2}\right)$. As control, the cells were incubated with DMEM containing DMSO $(0.5 \% \mathrm{v} / \mathrm{v})$ as a negative control or Triton ${ }^{\mathrm{TM}} \mathrm{X}-10(0.1 \%)$ as a positive control of cytotoxicity. After $24 \mathrm{~h}$ of dye incubation, the medium was replaced by 100 $\mu \mathrm{L}$ of MTT solution in DMEM and the cells were incubated for $3 \mathrm{~h}$ at $37^{\circ} \mathrm{C}$. Then, $75 \mu \mathrm{L}$ of the solution was replaced by $50 \mu \mathrm{L}$ of DMSO (100\%) and gently shaken for $15 \mathrm{~min}$ at room temperature. The absorbance at $540 \mathrm{~nm}$ was recorded. Each concentration of dye was tested in sextuplicate. For each concentration, we calculated the percentage of cell viability compared to the control DMEM+ $0.5 \%$ DMSO as $0 \mu \mathrm{M}$ of MCs.

\section{Confocal imaging and FLIM}

KB cells (ATCC ${ }^{\circledR}$ CCL-17) were grown in minimum essential medium (MEM, Gibco-Invitrogen) with $10 \%$ fetal bovine serum (FBS, Lonza), 1\% non-essential amino acids (Gibco-Invitrogen), 1\% MEM vitamin solution (Gibco-Invitrogen), 1\% L-Glutamine (Sigma Aldrich) and $0.1 \%$ antibiotic solution (gentamicin, Sigma-Aldrich) at $37^{\circ} \mathrm{C}$ in humidified atmosphere containing $5 \% \mathrm{CO}_{2}$. Cells were seeded onto a chambered coverglass $\left(I \mathrm{BiDi}^{\circledR}\right)$ at a density of $5 \times 10^{4}$ cells/well $24 \mathrm{~h}$ before the microscopy measurement. For imaging, the culture medium was removed and the attached cells were washed with OptiMEM (Gibco-Invitrogen). The live cells were stained with MC dyes $(1 \mu \mathrm{M})$ prior to imaging and were imaged without any washing steps. Nystatin was added from a stock solution of 1 $\mathrm{mM}$ in DMSO (final concentration used for cells: $5 \mu \mathrm{M}$ ) and cells were incubated for 30 minutes at $37^{\circ} \mathrm{C}$ in humidified atmosphere containing $5 \% \mathrm{CO}_{2}$ before addition of the dye. For the apoptosis induction, cells were incubated with staurosporine $\left(1 \mu \mathrm{M}\right.$ ) for 2 hours at $37^{\circ} \mathrm{C}$ (with $5 \% \mathrm{CO}_{2}$ ) prior to the dye treatment. The plasma membrane was stained with MemBright-488 (200 nM) prior to imaging. ${ }^{68}$ MitoTracker Green FM was purchased from ThermoFisher scientific. ${ }^{69}$ The colocalization studies were performed on several cells from different acquisitions using colocalization studio ${ }^{70}$ in the icy software. The images were all processed with Icy software. ${ }^{71}$

\section{Fluorescence lifetime imaging}

Lifetime imaging was performed using homemade two-photon excitation scanning fluorescence microscope based on an Olympus IX70 inverted microscope with an Olympus 60x 1.2NA water immersion objective operating in the descanned fluorescence collection mode. Two-photon excitation at 960 $\mathrm{nm}$ was provided by a mode-locked titanium-sapphire laser (Tsunami; Spectra Physics) or an Insight DeepSee (Spectra Physics) laser. We performed FLIM measurements using $30 \mu \mathrm{m}$ $\times 30 \mu \mathrm{m}$ scale and 128 pixels $\times 128$ pixels. All FLIM data were analyzed using a commercial software package (SPCImage v2.8; Becker \& Hickl, Germany).

\section{Conflicts of interest}

There are no conflicts of interest to declare

\section{Acknowledgements}

This work was supported by the French National Research Agency (ANR) BrightSwitch 19-CE29-0005-01, BrightRiboProbes ANR-16-CE11-0010-01/02 and the European Research Council (ERC) consolidator grant BrightSens 648528. TM and SK acknowledge IIT Gandhinagar and SERB, Govt. of India (CRG/2018/004020). The authors would also like to thank the analysis platform (PACSI).

\section{References}

1 (1) Luby-Phelps, K. Cytoarchitecture and Physical Properties of Cytoplasm: Volume, Viscosity, Diffusion, Intracellular Surface Area. In International Review of Cytology; Walter, H., Brooks, D. E., Srere, P. A., Eds.; Microcompartmentation and Phase Separation in Cytoplasm; Academic Press, 1999; Vol. 192, pp 189-221. https://doi.org/10.1016/S00747696(08)60527-6.

2 (2) Deliconstantinos, G.; Villiotou, V.; Stavrides, J. C. Modulation of Particulate Nitric Oxide Synthase Activity and Peroxynitrite Synthesis in Cholesterol Enriched Endothelial Cell Membranes. Biochem. Pharmacol. 1995, 49 (11), 15891600. https://doi.org/10.1016/0006-2952(95)00094-G.

3 (3) Nadiv, O.; Shinitzky, M.; Manu, H.; Hecht, D.; Roberts, C. T.; LeRoith, D.; Zick, Y. Elevated Protein Tyrosine Phosphatase Activity and Increased Membrane Viscosity Are Associated with Impaired Activation of the Insulin Receptor Kinase in Old Rats. Biochem. J. 1994, 298 (2), 443-450. https://doi.org/10.1042/bj2980443.

4 (4) Zubenko, G. S.; Kopp, U.; Seto, T.; Firestone, L. L. Platelet Membrane Fluidity Individuals at Risk for Alzheimer's Disease: A Comparison of Results from Fluorescence Spectroscopy and Electron Spin Resonance Spectroscopy. Psychopharmacology (Berl.) 1999, 145 (2), 175-180. https://doi.org/10.1007/s002130051046.

5 (5) Klymchenko, A. S. Solvatochromic and Fluorogenic Dyes as Environment-Sensitive Probes: Design and Biological Applications. Acc. Chem. Res. 2017, 50 (2), 366-375. https://doi.org/10.1021/acs.accounts.6b00517.

6 (6) Liu, T.; Liu, X.; Spring, D. R.; Qian, X.; Cui, J.; Xu, Z. Quantitatively Mapping Cellular Viscosity with Detailed Organelle Information via a Designed PET Fluorescent Probe. Sci. Rep. 2014, 4 (1), 1-7. https://doi.org/10.1038/srep05418.

7 (7) Liu, F.; Wen, J.; Chen, S.-S.; Sun, S. A Luminescent Bimetallic Iridium(III) Complex for Ratiometric Tracking Intracellular Viscosity. Chem. Commun. 2018, 54 (11), 13711374. https://doi.org/10.1039/C7CC09723A.

8 (8) Haidekker, M. A.; Theodorakis, E. A. Molecular RotorsFluorescent Biosensors for Viscosity and Flow. Org. Biomol. Chem. 2007, 5 (11), 1669-1678. https://doi.org/10.1039/b618415d.

9 (9) Haidekker, M. A.; Theodorakis, E. A. EnvironmentSensitive Behavior of Fluorescent Molecular Rotors. J. Biol. Eng. 2010, 4 (1), 11. https://doi.org/10.1186/1754-1611-411.

10 (10) Kuimova, M. K. Mapping Viscosity in Cells Using Molecular Rotors. Phys. Chem. Chem. Phys. PCCP 2012, 14 (37), 12671-12686. https://doi.org/10.1039/c2cp41674c.

11 (11) Lee, S.-C.; Heo, J.; Woo, H. C.; Lee, J.-A.; Seo, Y. H.; Lee, C.-L.; Kim, S.; Kwon, O.-P. Fluorescent Molecular Rotors for Viscosity Sensors. Chem. - Eur. J. 2018, 24 (52), 1370613718. https://doi.org/10.1002/chem.201801389. 
12 (12) Miao, W.; Yu, C.; Hao, E.; Jiao, L. Functionalized BODIPYs as Fluorescent Molecular Rotors for Viscosity Detection. Front. Chem. 2019 https://doi.org/10.3389/fchem.2019.00825.

13 (13) Raut, S.; Kimball, J.; Fudala, R.; Doan, H.; Maliwal, B.; Sabnis, N.; Lacko, A.; Gryczynski, I.; Dzyuba, S. V.; Gryczynski, Z. A Homodimeric BODIPY Rotor as a Fluorescent Viscosity Sensor for Membrane-Mimicking and Cellular Environments. Phys. Chem. Chem. Phys. 2014, 16 (48), 27037-27042. https://doi.org/10.1039/C4CP04260C.

14 (14) López-Duarte, I.; Vu, T. T.; Izquierdo, M. A.; Bull, J. A.; Kuimova, M. K. A Molecular Rotor for Measuring Viscosity in Plasma Membranes of Live Cells. Chem. Commun. 2014, 50 (40), 5282-5284. https://doi.org/10.1039/C3CC47530A.

15 (15) Song, X.; Li, N.; Wang, C.; Xiao, Y. Targetable and Fixable Rotor for Quantifying Mitochondrial Viscosity of Living Cells by Fluorescence Lifetime Imaging. J. Mater. Chem. B 2017, 5 (2), 360-368. https://doi.org/10.1039/C6TB02524B.

16 (16) Wang, L.; Xiao, Y.; Tian, W.; Deng, L. Activatable Rotor for Quantifying Lysosomal Viscosity in Living Cells. J. Am. Chem. Soc. 2013, 135 (8), 2903-2906 https://doi.org/10.1021/ja311688g.

17 (17) Chambers, J. E.; Kubánková, M.; Huber, R. G.; LópezDuarte, I.; Avezov, E.; Bond, P. J.; Marciniak, S. J.; Kuimova, M. K. An Optical Technique for Mapping Microviscosity Dynamics in Cellular Organelles. ACS Nano 2018, 12 (5), 4398-4407. https://doi.org/10.1021/acsnano.8b00177.

18 (18) Ashokkumar, P.; Ashoka, A. H.; Collot, M.; Das, A.; Klymchenko, A. S. A Fluorogenic BODIPY Molecular Rotor as an Apoptosis Marker. Chem. Commun. 2019, 55 (48), 69026905. https://doi.org/10.1039/C9CC03242H.

19 (19) Yuan, L.; Lin, W.; Zheng, K.; He, L.; Huang, W. Far-Red to near Infrared Analyte-Responsive Fluorescent Probes Based on Organic Fluorophore Platforms for Fluorescence Imaging. Chem. Soc. Rev. 2012, 42 (2), 622-661. https://doi.org/10.1039/C2CS35313J.

20 (20) Umezawa, K.; Citterio, D.; Suzuki, K. New Trends in NearInfrared Fluorophores for Bioimaging. Anal. Sci. 2014, 30 (3), 327-349. https://doi.org/10.2116/analsci.30.327.

21 (21) Qu, Y.; Zhu, Y.; Wu, J.; Wu, J.; Gu, Z.; Wu, Y. Molecular Rotor Based on Dipyridylphenylamine: Near-Infrared Enhancement Emission from Restriction of Molecular Rotation and Applications in Viscometer and Bioprobe. Dyes Pigments 2020, 172, 107795. https://doi.org/10.1016/j.dyepig.2019.107795.

22 (22) Li, S.; Wang, P.; Feng, W.; Xiang, Y.; Dou, K.; Liu, Z. Simultaneous Imaging of Mitochondrial Viscosity and Hydrogen Peroxide in Alzheimer's Disease by a Single nearInfrared Fluorescent Probe with a Large Stokes Shift. Chem. Commun. 2020, 56 (7), 1050-1053. https://doi.org/10.1039/C9CC08267K.

23 (23) Mudliar, N. H.; Singh, P. K. A Molecular Rotor-Based Turn-on Sensor Probe for Amyloid Fibrils in the Extreme near-Infrared Region. Chem. Commun. 2019, 55 (27), 39073910. https://doi.org/10.1039/C9CC01262A.

24 (24) Guo, R.; Ma, Y.; Tang, Y.; Xie, P.; Wang, Q.; Lin, W. A Novel Mitochondria-Targeted near-Infrared (NIR) Probe for Detection of Viscosity Changes in Living Cell, Zebra Fishes and Living Mice. Talanta 2019, 204, 868-874. https://doi.org/10.1016/j.talanta.2019.06.050.

25 (25) Ma, Y.; Zhao, Y.; Guo, R.; Zhu, L.; Lin, W. A Near-Infrared Emission Fluorescent Probe with Multi-Rotatable Moieties for Highly Sensitive Detection of Mitochondrial Viscosity in an Inflammatory Cell Model. J. Mater. Chem. B 2018, 6 (39), 6212-6216. https://doi.org/10.1039/C8TB02083C

26 (26) Hou, M.-X.; Liu, L.-Y.; Wang, K.-N.; Chao, X.-J.; Liu, R.-X.; Mao, Z.-W. A Molecular Rotor Sensor for Detecting Mitochondrial Viscosity in Apoptotic Cells by Two-Photon
Fluorescence Lifetime Imaging. New J. Chem. 2020, 44 (26), 11342-11348. https://doi.org/10.1039/D0NJ02108C.

27 (27) Peng, X.; Yang, Z.; Wang, J.; Fan, J.; He, Y.; Song, F.; Wang, B.; Sun, S.; Qu, J.; Qi, J.; Yan, M. Fluorescence Ratiometry and Fluorescence Lifetime Imaging: Using a Single Molecular Sensor for Dual Mode Imaging of Cellular Viscosity. J. Am. Chem. Soc. 2011, 133 (17), 6626-6635. https://doi.org/10.1021/ja1104014.

28 (28) Mu, X.; Liu, Y.; Liu, S.; Sun, Y.; Lu, N.; Lu, Y.; Li, W.; Zhou, X.; Liu, B.; Li, Z. A Cyanine-Derived near-Infrared Molecular Rotor for Ratiometric Imaging of Mitochondrial Viscosity in Cells. Sens. Actuators B Chem. 2019, 298, 126831. https://doi.org/10.1016/j.snb.2019.126831.

29 (29) Karpenko, I. A.; Niko, Y.; Yakubovskyi, V. P.; Gerasov, A. O.; Bonnet, D.; Kovtun, Y. P.; Klymchenko, A. S. Push-Pull Dioxaborine as Fluorescent Molecular Rotor: Far-Red Fluorogenic Probe for Ligand-Receptor Interactions. J. Mater. Chem. C 2016, 4 (14), 3002-3009. https://doi.org/10.1039/C5TC03411F.

30 (30) Guo, R.; Yin, J.; Ma, Y.; Li, G.; Wang, Q.; Lin, W. A Novel NIR Probe for Detection of Viscosity in Cellular Lipid Droplets, Zebra Fishes and Living Mice. Sens. Actuators $B$ Chem. 2018, 271, 321-328. https://doi.org/10.1016/j.snb.2018.05.055.

31 (31) Yan, J.; Zhu, J.; Zhou, K.; Wang, J.; Tan, H.; Xu, Z.; Chen, S.; Lu, Y.; Cui, M.; Zhang, L. Neutral Merocyanine Dyes: For in Vivo NIR Fluorescence Imaging of Amyloid- $\beta$ Plaques. Chem. Commun. 2017, 53 (71), 9910-9913. https://doi.org/10.1039/C7CC05056A.

32 (32) Yuan, L.; Lin, W.; Zheng, K.; He, L.; Huang, W. Far-Red to near Infrared Analyte-Responsive Fluorescent Probes Based on Organic Fluorophore Platforms for Fluorescence Imaging. Chem. Soc. Rev. 2012, 42 (2), 622-661. https://doi.org/10.1039/C2CS35313J.

33 (33) Liu, Y.; Teng, L.; Chen, L.; Ma, H.; Liu, H.-W.; Zhang, X.-B. Engineering of a Near-Infrared Fluorescent Probe for RealTime Simultaneous Visualization of Intracellular Hypoxia and Induced Mitophagy. Chem. Sci. 2018, 9 (24), 5347-5353. https://doi.org/10.1039/C8SC01684D.

34 (34) Chen, H.; Lin, W.; Cui, H.; Jiang, W. Development of Unique Xanthene-Cyanine Fused Near-Infrared Fluorescent Fluorophores with Superior Chemical Stability for Biological Fluorescence Imaging. Chem. - Eur. J. 2015, 21 (2), 733-745. https://doi.org/10.1002/chem.201404718.

35 (35) Yuan, L.; Lin, W.; Zhao, S.; Gao, W.; Chen, B.; He, L.; Zhu, S. A Unique Approach to Development of Near-Infrared Fluorescent Sensors for in Vivo Imaging. J. Am. Chem. Soc. 2012, 134 (32), 13510-13523. https://doi.org/10.1021/ja305802v.

36 (36) Li, Y.; Wang, Y.; Yang, S.; Zhao, Y.; Yuan, L.; Zheng, J.; Yang, R. Hemicyanine-Based High Resolution Ratiometric near-Infrared Fluorescent Probe for Monitoring PH Changes in Vivo. Anal. Chem. 2015, 87 (4), 2495-2503. https://doi.org/10.1021/ac5045498.

37 (37) Wan, Q.; Chen, S.; Shi, W.; Li, L.; Ma, H. Lysosomal PH Rise during Heat Shock Monitored by a Lysosome-Targeting Near-Infrared Ratiometric Fluorescent Probe. Angew. Chem. Int. Ed. 2014, 53 (41), 10916-10920. https://doi.org/10.1002/anie.201405742

38 (38) Zhu, S.; Lin, W.; Yuan, L. Development of a Near-Infrared Fluorescent Probe for Monitoring Hydrazine in Serum and Living Cells. Anal. Methods 2013, 5 (14), 3450-3453. https://doi.org/10.1039/C3AY40540K.

39 (39) Luo, Z.; Feng, L.; An, R.; Duan, G.; Yan, R.; Shi, H.; He, J.; Zhou, Z.; Ji, C.; Chen, H.-Y.; Ye, D. Activatable Near-Infrared Probe for Fluorescence Imaging of $Y$-Glutamyl Transpeptidase in Tumor Cells and In Vivo. Chem. - Eur. J. 
2017, 23

(59),

$14778-14785$

https://doi.org/10.1002/chem.201702210.

40 (40) Bou, S.; Wang, X.; Anton, N.; Klymchenko, A. S.; Collot, M. Near Infrared Fluorogenic Probe as a Prodrug Model for Evaluating Cargo Release by Nanoemulsions. J. Mater. Chem. $\begin{array}{llll}B & 2020, & \text { (27), 5938-5944. }\end{array}$ https://doi.org/10.1039/D0TB00783H.

41 (41) Collot, M.; Ponsot, F.; Klymchenko, A. S. Ca-NIR: A Ratiometric near-Infrared Calcium Probe Based on a Dihydroxanthene-Hemicyanine Fluorophore. Chem. Commun. 2017, 53 (45), 6117-6120. https://doi.org/10.1039/C7CC02418E.

42 (42) Romieu, A.; Richard, J.-A. An Expedient Synthesis of N,NDialkylamino-Dihydroxanthene-Pyrylium Conjugated nearInfrared Fluorescent Dyes. Tetrahedron Lett. 2016, 57 (3), 317-320. https://doi.org/10.1016/j.tetlet.2015.12.010.

43 (43) Ong, M. J. H.; Srinivasan, R.; Romieu, A.; Richard, J.-A. Divergent Synthesis of Dihydroxanthene-Hemicyanine Fused Near-Infrared Fluorophores through the Late-Stage Amination of a Bifunctional Precursor. Org. Lett. 2016, 18 (19), https://doi.org/10.1021/acs.orglett.6b02564.

44 (44) Ong, M. J. H.; Debieu, S.; Moreau, M.; Romieu, A.; Richard, J.-A. Synthesis of N,N-Dialkylamino-norDihydroxanthene-Hemicyanine Fused Near-Infrared Fluorophores and Their First Water-Soluble and/or Bioconjugatable Analogues. Chem. - Asian J. 2017, 12 (8), 936-946. https://doi.org/10.1002/asia.201700176.

45 (45) Tong, Z.-X.; Liu, W.; Huang, H.; Chen, H.-Z.; Liu, X.-J. Kuang, Y.-Q.; Jiang, J.-H. A Ratiometric Fluorescent PH Probe Based on Keto-Enol Tautomerization for Imaging of Living Cells in Extreme Acidity. Analyst 2017, 142 (20), 3906-3912. https://doi.org/10.1039/C7AN01103B.

46 (46) Easton, T. G.; Valinsky, J. E.; Reich, E. Merocyanine 540 as a Fluorescent Probe of Membranes: Staining of Electrically Excitable Cells. Cell 1978, 13 (3), 475-486. https://doi.org/10.1016/0092-8674(78)90321-5.

47 (47) Kulinich, A. V.; Derevyanko, N. A.; Mikitenko, E. K.; Ishchenko, A. A. Merocyanines Based on 1,3-Indanedione: Electronic Structure and Solvatochromism. J. Phys. Org. Chem. 2011, 24 (8), 732-742. https://doi.org/10.1002/poc.1821.

48 (48) Collot, M.; Fam, T. K.; Ashokkumar, P.; Faklaris, O.; Galli, T.; Danglot, L.; Klymchenko, A. S. Ultrabright and Fluorogenic Probes for Multicolor Imaging and Tracking of Lipid Droplets in Cells and Tissues. J. Am. Chem. Soc. 2018, 140 (16), 54015411. https://doi.org/10.1021/jacs.7b12817.

49 (49) Fam, T.; Klymchenko, A.; Collot, M. Recent Advances in Fluorescent Probes for Lipid Droplets. Materials 2018, 11 (9), 1768. https://doi.org/10.3390/ma11091768.

50 (50) Collot, M.; Bou, S.; Fam, T. K.; Richert, L.; Mély, Y.; Danglot, L.; Klymchenko, A. S. Probing Polarity and Heterogeneity of Lipid Droplets in Live Cells Using a PushPull Fluorophore. Anal. Chem. 2019, 91 (3), 1928-1935. https://doi.org/10.1021/acs.analchem.8b04218.

51 (51) Ashoka, A. H.; Ashokkumar, P.; Kovtun, Y. P.; Klymchenko, A. S. Solvatochromic Near-Infrared Probe for Polarity Mapping of Biomembranes and Lipid Droplets in Cells under Stress. J. Phys. Chem. Lett. 2019, 10 (10), 24142421. https://doi.org/10.1021/acs.jpclett.9b00668.

52 (52) Guo, R.; Yin, J.; Ma, Y.; Li, G.; Wang, Q.; Lin, W. A Novel NIR Probe for Detection of Viscosity in Cellular Lipid Droplets, Zebra Fishes and Living Mice. Sens. Actuators $B$ Chem. 2018, 271, 321-328. https://doi.org/10.1016/j.snb.2018.05.055.

53 (53) Wang, X.; Anton, N.; Ashokkumar, P.; Anton, H.; Fam, T. K.; Vandamme, T.; Klymchenko, A. S.; Collot, M. Optimizing the Fluorescence Properties of Nanoemulsions for Single
Particle Tracking in Live Cells. ACS Appl. Mater. Interfaces 2019, 11 https://doi.org/10.1021/acsami.8b22297.

54 (54) Yang, Z.; He, Y.; Lee, J.-H.; Park, N.; Suh, M.; Chae, W.-S.; Cao, J.; Peng, X.; Jung, H.; Kang, C.; Kim, J. S. A SelfCalibrating Bipartite Viscosity Sensor for Mitochondria. J. Am. Chem. Soc. 2013, 135 (24), 9181-9185. https://doi.org/10.1021/ja403851p.

55 (55) Li, S.; Wang, P.; Feng, W.; Xiang, Y.; Dou, K.; Liu, Z. Simultaneous Imaging of Mitochondrial Viscosity and Hydrogen Peroxide in Alzheimer's Disease by a Single nearInfrared Fluorescent Probe with a Large Stokes Shift. Chem. $\begin{array}{llll}\text { Commun. 2020, } 56 & \text { (7), 1050-1053. }\end{array}$ https://doi.org/10.1039/C9CC08267K.

56 (56) Yin, J.; Peng, M.; Lin, W. Visualization of Mitochondrial Viscosity in Inflammation, Fatty Liver, and Cancer Living Mice by a Robust Fluorescent Probe. Anal. Chem. 2019, 91 (13) 8415-8421. https://doi.org/10.1021/acs.analchem.9b01293.

57 (57) Zielonka, J.; Joseph, J.; Sikora, A.; Hardy, M.; Ouari, O.; Vasquez-Vivar, J.; Cheng, G.; Lopez, M.; Kalyanaraman, B. Mitochondria-Targeted Triphenylphosphonium-Based Compounds: Syntheses, Mechanisms of Action, and Therapeutic and Diagnostic Applications. Chem. Rev. 2017 $117 \quad(15)$, https://doi.org/10.1021/acs.chemrev.7b00042.

58 (58) Yang, Z.; Cao, J.; He, Y.; Yang, J. H.; Kim, T.; Peng, X.; Kim, J. S. Macro-/Micro-Environment-Sensitive Chemosensing and Biological Imaging. Chem. Soc. Rev. 2014, 43 (13), 45634601. https://doi.org/10.1039/C4CS00051J.

59 (59) Belmokhtar, C. A.; Hillion, J.; Ségal-Bendirdjian, E. Staurosporine Induces Apoptosis through Both CaspaseDependent and Caspase-Independent Mechanisms. Oncogene 2001, $20 \quad$ (26), 3354-3362. https://doi.org/10.1038/sj.onc.1204436.

60 (60) Hu, Q.; Gao, M.; Feng, G.; Chen, X.; Liu, B. A Cell Apoptosis Probe Based on Fluorogen with Aggregation Induced Emission Characteristics. ACS Appl. Mater. Interfaces 2015, $\quad 7 \quad$ (8), $4875-4882$. https://doi.org/10.1021/am508838z.

61 (61) Chen, B.; Li, C.; Zhang, J.; Kan, J.; Jiang, T.; Zhou, J.; Ma, $\mathrm{H}$. Sensing and Imaging of Mitochondrial Viscosity in Living Cells Using a Red Fluorescent Probe with a Long Lifetime. Chem. Commun. 2019, 55 (51), 7410-7413. https://doi.org/10.1039/C9CC03977E.

62 (62) Hao, L.; Li, Z.-W.; Zhang, D.-Y.; He, L.; Liu, W.; Yang, J.; Tan, C.-P.; Ji, L.-N.; Mao, Z.-W. Monitoring Mitochondrial Viscosity with Anticancer Phosphorescent $\operatorname{Ir}($ III) Complexes via Two-Photon Lifetime Imaging. Chem. Sci. 2019, 10 (5), 1285-1293. https://doi.org/10.1039/C8SC04242J.

63 (63) Lakowicz, J. R. Principles of Fluorescence Spectroscopy; Springer Science \& Business Media, 2007.

64 (64) Alessi, A.; Salvalaggio, M.; Ruzzon, G. Rhodamine 800 as Reference Substance for Fluorescence Quantum Yield Measurements in Deep Red Emission Range. J. Lumin. 2013, 134, 385-389. https://doi.org/10.1016/j.jlumin.2012.08.017.

65 (65) Enderlein, J.; Erdmann, R. Fast Fitting of MultiExponential Decay Curves. Opt. Commun. 1997, 134 (1), 371-378. https://doi.org/10.1016/S0030-4018(96)00384-7.

66 (66) Smith, D. A.; McKenzie, G.; Jones, A. C.; Smith, T. A. Analysis of Time-Correlated Single Photon Counting Data: A Comparative Evaluation of Deterministic and Probabilistic Approaches. Methods Appl. Fluoresc. 2017, 5 (4), 042001. https://doi.org/10.1088/2050-6120/aa8055.

67 (67) Brochon, J.-C. [13] Maximum Entropy Method of Data Analysis in Time-Resolved Spectroscopy. In Methods in Enzymology; Part B: Numerical Computer Methods; Academic Press, 1994; Vol. 240, pp 262-311. https://doi.org/10.1016/S0076-6879(94)40052-0. 
68 (68) Collot, M.; Boutant, E.; Lehmann, M.; Klymchenko, A. S. BODIPY with Tuned Amphiphilicity as a Fluorogenic Plasma Membrane Probe. Bioconjug. Chem. 2019, 30 (1), 192-199. https://doi.org/10.1021/acs.bioconjchem.8b00828.

69 (69) Life Technologies https://www.thermofisher.com/.

70 (70) Lagache, T.; Sauvonnet, N.; Danglot, L.; Olivo-Marin, J.-C. Statistical Analysis of Molecule Colocalization in Bioimaging. Cytometry A 2015, 87 (6), 568-579. https://doi.org/10.1002/cyto.a.22629.
71 (71) Chaumont, F. de; Dallongeville, S.; Chenouard, N.; Hervé, N.; Pop, S.; Provoost, T.; Meas-Yedid, V.; Pankajakshan, P.; Lecomte, T.; Montagner, Y. L.; Lagache, T.; Dufour, A.; Olivo-Marin, J.-C. Icy: An Open Bioimage Informatics Platform for Extended Reproducible Research. Nat. Methods 2012, 9 (7), nmeth.2075. https://doi.org/10.1038/nmeth.2075.

Table of content

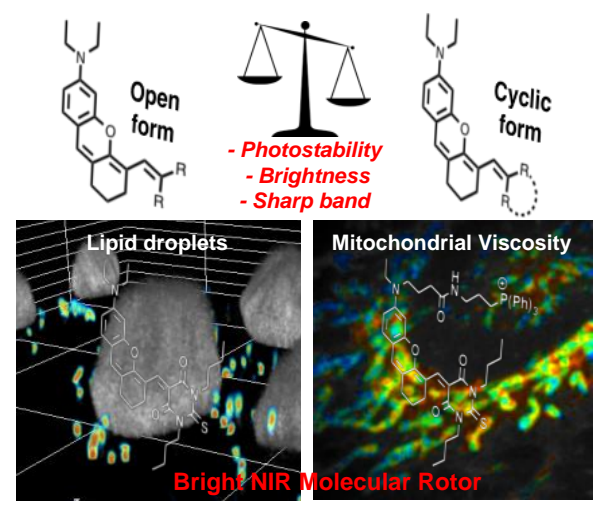

